\title{
Remote FxLMS Algorithm for Active Control of Sound in Remote Locations
}

\author{
Iman Tabatabaei Ardekani* ${ }^{*}$, Waleed H. Abdulla ${ }^{\dagger}$ and Saeed Ur Rehman* \\ * Computing Dept., UNITEC Institute of Technology, Auckland, New Zealand \\ $\dagger$ Electrical and Computer Eng. Dept., The University of Auckland, Auckland, New Zealand
}

\begin{abstract}
Available adaptive control algorithms can only create a silent point at the locations of an error microphone. In this case, the error microphone occupies a part of the zone of quiet established around the silent point, resulting in an ineffective use of space in the zone of quiet. This paper develops a novel adaptive control algorithm for active control of sound in remote locations rather than the locations of error microphones. It establishes a link between the performance of the FxLMS adaptive algorithm in the digital control domain and the performance of the active noise control mechanism in the acoustic domain. The transfer function of an optimal controller that can minimize the acoustic pressure in a location away from the error microphone is first derived. For the realization of the proposed controller, an adaptive algorithm, called Remote FxLMS is then developed. Different computer simulations are used to show the efficiency of the proposed algorithm.
\end{abstract}

\section{INTRODUCTION}

Active noise control (ANC) has been paid attention in the control of low frequency sound fields [1]. It has a multidisciplinary nature combining acoustics and digital signal processing. In view point of acoustics, ANC is formulated in the field theory context [2] and it concerns finding a secondary (control) field that can shape, control, or cancel a primary (disturbance) field at a certain point. Here, applying wave propagation equations set results in a general solution; however, this solution can only apply to ideal cases with deterministic accurate models [3]-[5]. In digital signal processing, ANC can be formulated as an adaptive system identification framework. The solutions obtained from this approach are particularly referred to as adaptive ANC [6].

The first attempts to apply adaptive signal processing techniques for developing ANC systems were reported by Burgess [7] and Warnaka [8], independently. Burgess derived the Filtered-x Least Mean Square (FxLMS) algorithm as the key component of ANC systems. Widrow derived a similar algorithm in the context of adaptive control for adaptive inverse control problems [9]. The FxLMS algorithm can be derived by modifying the well-known Least-Mean-Square algorithm. This algorithm considers the power of an error signal (measured by a microphone called the error microphone) as a cost function. Then, it estimates and generates a control signal adaptively. The control signal is fed to a loudspeaker. The combination of the original noise field and the sound filed established by the loudspeaker makes a silent point at the location of the error microphone. The FxLMS algorithm is a computationally efficient algorithm that has been found very successful in ANC problems. However, its performance analysis is associated with high level of complexity. The authors have extensively studied FxLMS algorithm performance [10]-[14], resulting in the identification of available restrictions. One of the main restrictions of available FxLMS-based ANC systems is that they are only able to control the acoustic pressure at the location of an error microphone. A limited zone of quiet is then produced around the microphone as a by-product.

The main motivation of this paper is to develop a new adaptive control system that can minimize the acoustic pressure at a location different from the error microphone. In this case, the effective space of the zone of quiet can be extended, as this zone is not occupied by any microphone. A new adaptive algorithm, called the Remote FxLMS, is developed as the key component of the proposed active noise control system.

\section{FXLMS-BASED ANC}

Fig. 1 shows the functional block diagram of single-channel FxLMS-based ANC, where the sound pressure at the location of a single error microphone is adaptively controlled (or minimized). Hardware components of the system include two microphones, called reference and error microphones, a loudspeaker called control (or secondary) source and a digital control system for the implementation of the adaptive filter $W$ and its update mechanism (FxLMS algorithm). In this diagram, the reference signal $u(n)$ is picked up by the the reference microphone, the error signal $e(n)$ is picked up by the error microphone, and the anti-noise signal $v(n)$ is produced by the control source. The digital control system is responsible for the real-time estimation and generation of $v(n)$. Linear system, $G$ represents ambient influences on noise from the reference microphone to the error microphone and $H$ represents ambient influences on anti-noise from the control source to the error microphone. $G$ and $H$ are respectively called primary and secondary paths in ANC literature.

\section{A. FxLMS Algorithm}

For an ideal ANC controller, the acoustic pressure at the location of the error microphone is zero: $e(n)=0$. From the block diagram, the optimal controller for satisfying $e(n)=0$ should be set to

$$
W_{\text {opt }}(z)=-\frac{G(z)}{H(z)}
$$

Here, the main problem is that $G$ and $H$ are unknown systems. Even if we can reach an estimate of $H$, its inverse can be 


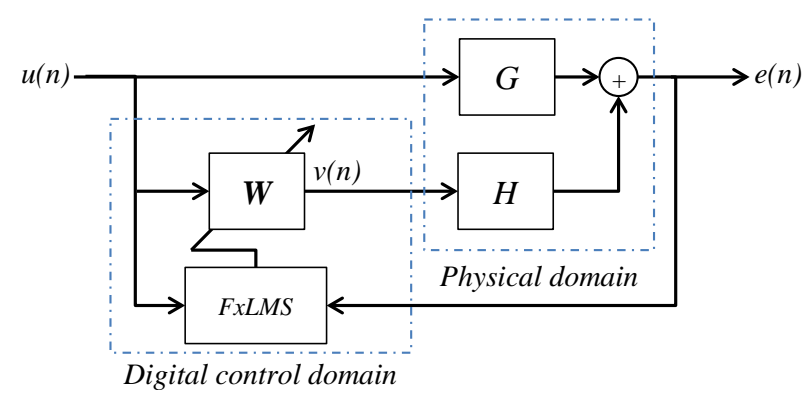

Figure 1. block diagram of FxLMS based ANC

unstable and non-causal. For solving this problem, the FxLMS algorithm is used to adjust $W$ adaptively, until it converges to a stable and causal solution for $W_{\text {opt }}$. Concurrently, the acoustic pressure at the location of the error microphone converges to a minimal level, as the FxLMS algorithm uses the power of $e(n)$ as a cost function. The FxLMS algorithm is given by [9]

$$
\mathbf{w}(n+1)=\mathbf{w}(n)+\mu e(n) \mathbf{u}_{H}(n)
$$

where $\mu$ is the adaptation step-size and $\mathbf{w}$ is a vector of length $L$, formed by the impulse response coefficients of $W$ :

$$
\mathbf{w}(n)=\left[\begin{array}{llll}
w_{0}(n) & w_{1}(n) & \ldots & w_{L-1}(n)
\end{array}\right]^{T}
$$

$\mathbf{u}_{H}(n)$ is calculated by filtering a tap vector of the reference signal, $\mathbf{u}(n)$, using an estimate of $H$. Assuming that an accurate estimate of $H$ is available, $\mathbf{u}_{H}(n)$ can be formulated by

$$
\mathbf{u}_{H}(n)=\sum_{q=0}^{Q-1} h_{q} \mathbf{u}(n-q)
$$

where $h_{0}, h_{1}, \ldots, h_{Q-1}$ are impulse response coefficients of $H$ and $\mathbf{u}(n)$ is given by:

$$
\mathbf{u}(n)=\left[\begin{array}{llll}
u(n) & u(n-1) & \ldots & u(n-L+1)
\end{array}\right]^{T}
$$

After updating $W$ by Eq. (2), the anti-noise signal $v(n)$ is computed as the response of $W$ to $u(n)$ :

$$
v(n)=\mathbf{w}(n)^{T} \mathbf{u}(n)
$$

The control source is fed by $v(n)$ to generate anti-noise in the acoustic domain. The combination of the sound fields generated by the original noise and anti-noise causes the acoustic pressure at the location of the error microphone to be minimal. In the following an optimal solution for $W$ is formulated in terms of physical variables.

\section{B. Optimal ANC Controller}

To study the performance of FxLMS-based ANC in the acoustic domain, a different analysis has to be performed as it is needed to formulate ANC behaviors in terms of physical variables. For this purpose, the locations of the ANC components have to be formulated in a coordinate system. Initially, we assume that the error microphone, the noise source and the reference microphone are located in three arbitrary locations of $\mathrm{L}_{0}, \mathrm{~L}_{1}$ and $\mathrm{L}_{2}$. Here, a coordinate system can be defined in such a way that

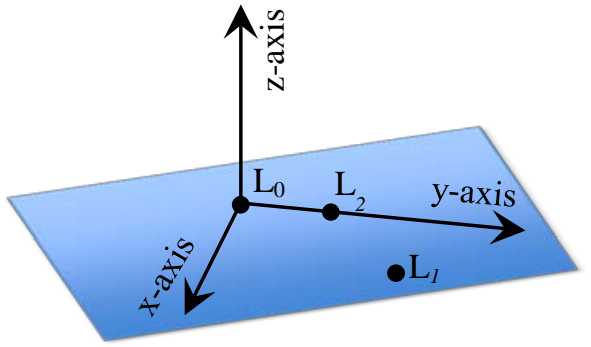

Figure 2. arbitrary locations of ANC components

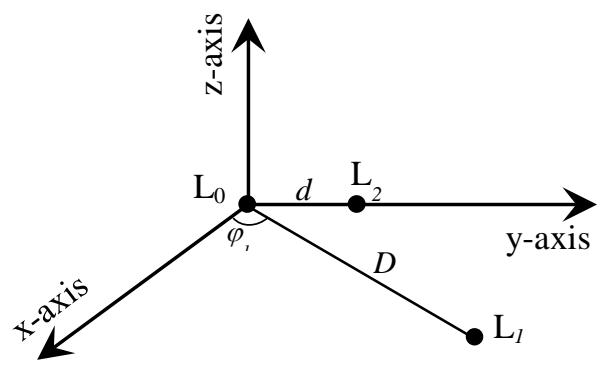

Figure 3. ANC components locations in a coordinate system

1) $\mathrm{L}_{0}$ is located at the origin of the coordinate system.

2) $L_{2}$ is located at the $y$-axis of the coordinate system.

3) The plane containing $L_{0}, L_{1}$ and $L_{2}$ forms the $x-y$ axis of the coordinate system.

The above-mentioned coordinate system is shown in Figs. 2 and 3. As shown in Fig. 3, the distance between the reference microphone and the error microphone is shown by $D=\mathrm{L}_{0} \mathrm{~L}_{1}$ and the distance between the control source and the error microphone is shown by $d=\mathrm{L}_{0} \mathrm{~L}_{2}$.

The acoustic pressure at the origin (error signal) is initially expressed by the the combination of the sound pressure caused by noise $u(n)$ and the one caused by anti-noise $v(n)$ :

$$
e(n)=\frac{1}{D} f_{1}(n) \circledast u\left(n-\frac{f_{s}}{c} D\right)+\frac{1}{d} f_{2}(n) \circledast v\left(n-\frac{f_{s}}{c} d\right)
$$

where $f_{s}$ is the sampling frequency, $c$ is the sound velocity in the ambient, $\circledast$ denotes the linear convolution operator and where $f_{1}(n)$ and $f_{2}(n)$ are two unknown transfer functions modeling possible reflections, reverberations and any other uncertainties associated with the sound propagation in the ambient. From Eq. (7), one can find the following expression for the error signal in z-domain:

$$
E(z)=\frac{1}{D} F_{1}(z) U(z) z^{-\frac{D}{c_{s}}}+\frac{1}{d} F_{2}(z) V(z) z^{-\frac{d}{c_{s}}}
$$

where $E(z), U(z), V(z), F_{1}(z)$ and $F_{2}(z)$ map $e(n), u(n)$, $v(n), f_{1}(n)$ and $f_{2}(n)$ into the z-domain respectively and $c_{s}$ is given by

$$
c_{s}=\frac{c}{f_{s}}
$$

Now by setting $E(z)=0$ in Eq. (8), one can obtain the following response for $V(z)$ :

$$
\left.V(z)\right|_{E(z)=0}=-\frac{d}{D} \frac{F_{1}(z)}{F_{2}(z)} z^{-\left(\frac{D-d}{c_{s}}\right)} U(z)
$$




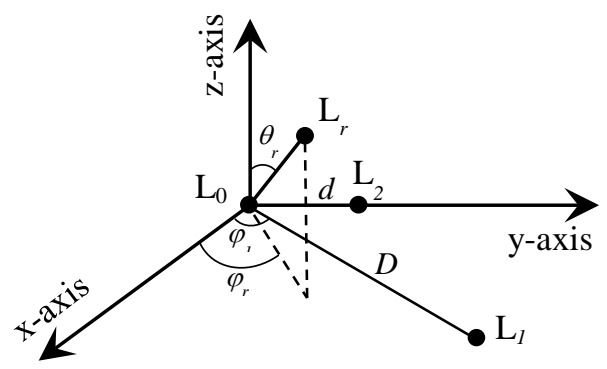

Figure 4. Remote ANC components locations in a coordinate system

From the block diagram, $\frac{V(z)}{U(z)}$ can be considered as the transfer function of the ANC controller. Accordingly, the optimal ANC controller can be formulated from Eq. (11) by

$$
W_{\text {opt }}(z)=-\frac{d}{D} \frac{F_{1}(z)}{F_{2}(z)} z^{-\left(\frac{D-d}{c_{s}}\right)}
$$

The direct implementation of the controller given in Eq. (11) is not technically possible because it requires geometrical parameters and acoustical characteristics of the physical plant, i.e. $F_{1}(z)$ and $F_{2}(z)$, to be known. However, this formulation corresponds to the optimal solution obtained by using the FxLMS algorithm. In fact, Eq. (11) gives an alternative expression for the optimal solution of the FxLMS algorithm in terms of physical parameters in the acoustic domain.

\section{ANC IN REMOTE LOCATIONS}

The proposed remote ANC system considers that the error microphone is still located at the origin $\mathrm{L}_{0}$ but the ANC system minimizes the acoustic pressure at a location far from the error microphone. Let us assume that the location of the desired remote silent point is shown by $\mathrm{L}_{r}$. The location of $\mathrm{L}_{r}$ along with the locations of the error microphone, reference microphone and the control source are shown in Fig. 4. According to this figure, $r, \theta_{r}$ and $\varphi_{r}$ show the radial distance, polar angle and Azimuthal angle of $\mathrm{L}_{r}$.

\section{A. Optimal ANC Controller for Remote Locations}

The acoustic pressure at $\mathrm{L}_{r}$ (remote silent point) is formulated by

$e_{r}(n)=\frac{1}{d_{1 r}} f_{1 r}(n) \circledast u\left(n-\frac{d_{1 r}}{c_{s}}\right)+\frac{1}{d_{2 r}} f_{2 r}(n) \circledast v\left(n-\frac{d_{2 r}}{c_{s}}\right)$

where $d_{1 r}$ and $d_{2 r}$ is the distance of $\mathrm{L}_{r}$ from the reference microphone $\left(\mathrm{L}_{1}\right)$ and control source $\left(\mathrm{L}_{2}\right)$, respectively and where $f_{1 r}(n)$ and $f_{2 r}(n)$ are two unknown transfer functions modeling possible reflections, reverberations and any other uncertainties associated with the sound propagation from $\mathrm{L}_{r}$ to $\mathrm{L}_{1}$ and $\mathrm{L}_{2}$. Assuming that $r$ is smaller than the noise wavelength, one can approximate $f_{1 r}(n) \approx f_{1}(n)$ and $f_{2 r}(n) \approx f_{2}(n)$; thus

$e_{r}(n)=\frac{1}{d_{1 r}} f_{1}(n) \circledast u\left(n-\frac{d_{1 r}}{c_{s}}\right)+\frac{1}{d_{2 r}} f_{2}(n) \circledast v\left(n-\frac{d_{2 r}}{c_{s}}\right)$

From Eq. (13), the acoustic pressure at $\mathrm{L}_{r}$ can be mapped to the $\mathrm{z}$-domain by

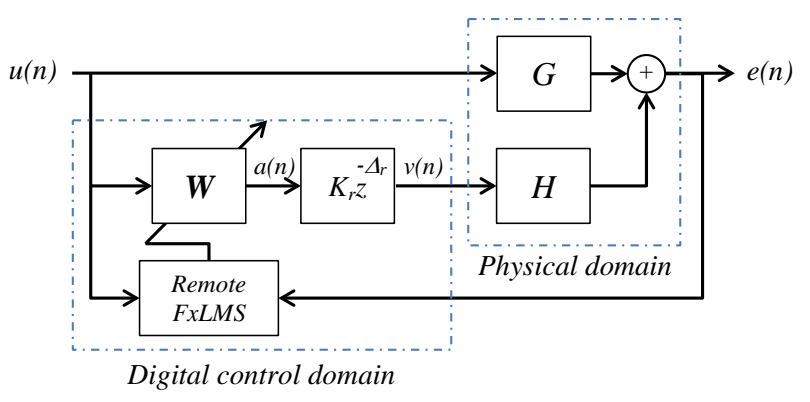

Figure 5. block diagram of ANC with remote FxLMS algorithm

$$
E_{r}(z)=\frac{1}{d_{1 r}} F_{1}(z) U(z) z^{-\frac{d_{1 r}}{c_{s}}}+\frac{1}{d_{2 r}} F_{2}(z) V(z) z^{-\frac{d_{2 r}}{c_{s}}}
$$

Now by setting $E_{r}(z)=0$ in Eq. (14), one can obtain

$$
\left.V_{r}(z)\right|_{E(z)=0}=-\frac{d_{2 r}}{d_{1 r}} \frac{F_{1}(z)}{F_{2}(z)} z^{-\left(\frac{d_{1 r}-d_{2 r}}{c_{s}}\right)} U(z)
$$

Therefore, the optimal controller for creating silence at $\mathrm{L}_{r}$ is

$$
\hat{W}_{\text {opt }}(z)=-\frac{d_{2 r}}{d_{1 r}} \frac{F_{1}(z)}{F_{2}(z)} z^{-\left(\frac{d_{1 r}-d_{2 r}}{c_{s}}\right)}
$$

From Fig. $4, d_{1 r}$ and $d_{2 r}$ can be formulated by

$$
d_{1 r}=\sqrt{D^{2}+r^{2}-2 r D \sin \theta_{r} \cos \left(\varphi_{r}-\varphi_{1}\right)}
$$

and

$$
d_{2 r}=\sqrt{r^{2}+d^{2}-2 r d \sin \theta_{r} \sin \varphi_{r}}
$$

For $r \ll D$ and $r \ll d$,

$$
d_{1 r} \approx D-r \sin \theta_{r} \cos \left(\varphi_{r}-\varphi_{1}\right)
$$

and

$$
d_{2 r} \approx d-r \sin \theta_{r} \sin \varphi_{r}
$$

Now, $\frac{d_{2 r}}{d_{1 r}}$ can be formulated by

$$
\frac{d_{2 r}}{d_{1 r}}=\frac{d}{D} \times \frac{1-\frac{r}{d} \sin \theta_{r} \sin \varphi_{r}}{1-\frac{r}{D} \sin \theta_{r} \cos \left(\varphi_{r}-\varphi_{1}\right)}
$$

For $r \ll D$,

$$
\frac{d_{2 r}}{d_{1 r}} \approx \frac{d}{D}\left(1-\frac{r}{d} \sin \theta_{r} \sin \varphi_{r}+\frac{r}{D} \sin \theta_{r} \cos \left(\varphi_{r}-\varphi_{1}\right)\right)
$$

Similarly, $d_{1 r}-d_{2 r}$ can be formulated by

$d_{1 r}-d_{2 r} \approx D-d-r \sin \theta_{r} \cos \left(\varphi_{r}-\varphi_{1}\right)+r \sin \theta_{r} \sin \varphi_{r}$

Combining Eqs. (16), (22) and (23) results in

$$
\hat{W}_{\text {opt }}(z)=K_{r} z^{-\triangle_{r}} W_{\text {opt }}(z)
$$

where $W_{\text {opt }}(z)$ is given in Eq. (11) and

$$
\begin{gathered}
K_{r}=1-\frac{r}{d} \sin \theta_{r} \sin \varphi_{r}+\frac{r}{D} \sin \theta_{r} \cos \left(\varphi_{r}-\varphi_{1}\right) \\
\triangle_{r}=r \sin \theta_{r}\left(\cos \left(\varphi_{r}-\varphi_{1}\right)-\sin \varphi_{r}\right)
\end{gathered}
$$

According to Eq. (24), $\hat{W}_{\text {opt }}(z)$ can be constructed by the series combination of a simple controller given by $K_{r} z^{-\triangle_{r}}$ 
and $W_{\text {opt }}(z)$ as shown in Fig. 5. In traditional ANC (shown in Fig. 1), FxLMS algorithm can reach an estimate of $W_{\text {opt }}(z)$; however, in the proposed ANC system $K_{r} z^{-\triangle_{r}}$ can affect on the adaptation process. In this case, the FxLMS algorithm cannot reach to $W_{\text {opt }}(z)$. Here, it is needed to modify the FxLMS algorithm so that it can compensate for the effects of $K_{r} z^{-\triangle_{r}}$ on the adaptation process. As shown in the block diagram given in Figs. 1 and 5, the transfer function $K_{r} z^{-\triangle_{r}}$ has no effects on the reference signal $u(n)$; however it can affect on $e(n)$. Thus, the effect of $K_{r} z^{-\triangle_{r}}$ on $e(n)$ should be compensated for. Integration of such compensation mechanism into the FxLMS algorithm leads to derive a new adaptive ANC algorithm, called Remote FxLMS. The traditional ANC algorithm can create a silent point at the location of the error microphone $\left(\mathrm{L}_{0}\right)$ but the proposed Remote FxLMS algorithm can create a silent point located at $\mathrm{L}_{r}$ rather than $\mathrm{L}_{0}$.

\section{B. Remote FxLMS Algorithm}

From Fig. 5, the available error signal in the Remote ANC system can be expressed by

$$
E(z)=G(z) U(z)+K_{r} z^{-\triangle_{r}} W(z) H(z) U(z)
$$

However, the desired error signal can be expressed by (obtained from Fig. 1)

$$
E^{*}(z)=G(z) U(z)+W(z) H(z) U(z)
$$

The difference between the desired and available error signals is

$$
\begin{aligned}
& \triangle E(z)=E^{*}(z)-E(z) \\
& =W(z) H(z) U(z)-K_{r} z^{-\triangle_{r}} W(z) H(z) U(z)
\end{aligned}
$$

From Fig. 5,

$$
A(z)=W(z) U(z)
$$

and

$$
V(z)=K_{r} z^{-\triangle_{r}} W(z) U(z)
$$

Combining Eqs. (29) and (30)-(31) results in

$$
\triangle E(z)=H(z)\{A(z)-V(z)\}
$$

which can be expressed in the time-domain as follows

$$
\triangle e(n)=\sum_{q=0}^{Q-1} h_{q}\{a(n-q)-v(n-q)\}
$$

where $h_{0}, h_{1}, \ldots, h_{Q-1}$ are the impulse response coefficients of $H . \triangle e(n)$ is the difference between the available error signal in the remote ANC system and the desired error signal. This difference should be added to the available error signal before being used by an FxLMS-like update equation. In this case, the new update equation of the Remote FxLMS algorithm can be obtained by modifying Eq. (2) to

$$
\mathbf{w}(n+1)=\mathbf{w}(n)+\mu\{e(n)+\triangle e(n)\} \mathbf{u}_{H}(n)
$$

This update equation can adaptively identify an estimate of $W_{\text {opt }}(z)$ at the presence of $K_{r} z^{-\triangle_{r}}$ in the control system.

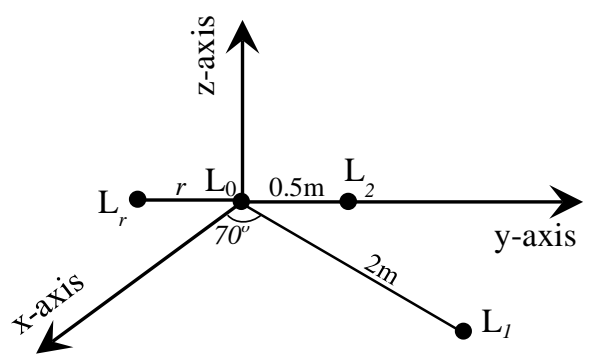

Figure 6. location of the ANC system components in the computer simulation
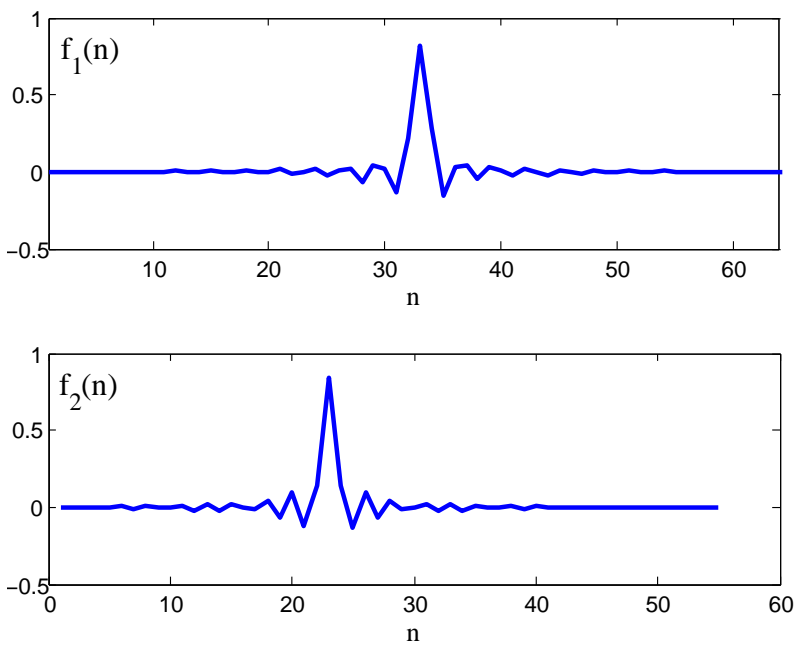

Figure 7. functions $f_{1}(n)$ and $f_{2}(n)$ in the computer simulation

\section{Simulation Results}

In order to show the validity of the theoretical results discussed above a set of computer simulations has been performed. The location of the noise source $\left(\mathrm{L}_{1}\right)$, control source $\left(\mathrm{L}_{2}\right)$, error microphone $\left(\mathrm{L}_{0}\right)$ and the remote silent point $\left(\mathrm{L}_{r}\right)$ have been shown in Fig. 6. The simulation is repeated for three different values of $r: 0 \mathrm{~cm}, 2 \mathrm{~cm}$ and $4 \mathrm{~cm}$. The impulse responses of $f_{1}$ and $f_{2}$ are also shown in Fig. 7. These responses represents the environmental influences on acoustic signal from $\mathrm{L}_{1}$ to $\mathrm{L}_{r}$ and from $\mathrm{L}_{2}$ to $\mathrm{L}_{r}$, respectively.

The system model used in the computer simulation contains the block diagram shown in Figs. 1 or 5. The reference signal $u(n)$ is a normalized broad-band white noise generated by the computer. The transfer functions of $G$ and $H$ can be obtained from Eq. (7) and Fig. 5 as

$$
G(z)=\frac{1}{D} F_{1}(z) z^{-\frac{D}{c_{s}}}
$$

and

$$
H(z)=\frac{1}{d} F_{2}(z) z^{-\frac{d}{c_{s}}}
$$

where $F_{1}$ and $F_{2}$ are the z-transforms of $f_{1}$ and $f_{2}$. The adaptive controller $W$ is updated by using Eqs. (2)-(5). The error signal (error microphone output) can be then calculated from the block diagram given in Figs. 1 or 4 . The acoustic pressure at the locations of the remote silent-point is calculated by using Eqs. (7) and (13).

In the first experiment, the traditional FxLMS algorithm is considered $(r=0)$. In this case, the silent point is 

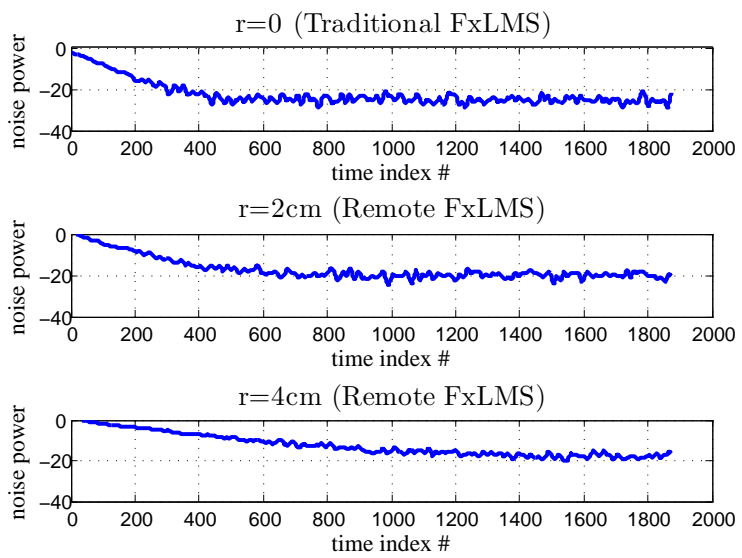

Figure 8 . noise power at the (remote) silent point

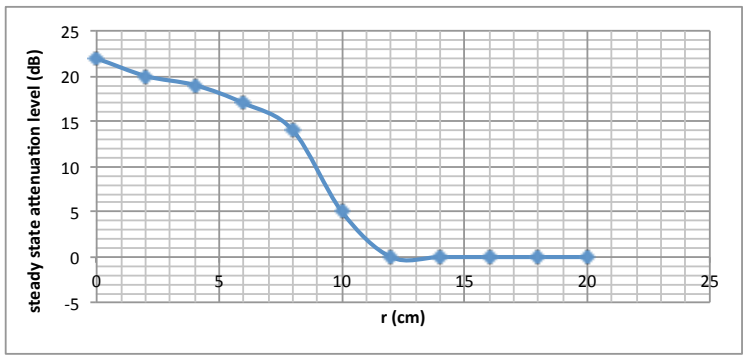

Figure 9. attenuation level of Remote-FxLMS algorithm for different distances between error microphone and remote silent point

located at the location of the error microphone. The power of the error signal is shown in Fig. 8. The noise power is estimated by averaging over 100 independent simulation runs. As seen, the noise is attenuated by about $22 \mathrm{~dB}$ in steadystate conditions. In the next experiment, the proposed Remote FxLMS algorithm is used and the remote silent-point is set $2 \mathrm{~cm}$ away from the error microphone. The noise power at the remote location is calculated by Eq. (13). After averaging over 100 independent experiments, the result is plotted in Fig. 8. As seen, the noise at the remote location is attenuated by about $20 \mathrm{~dB}$ in steady-state conditions. Compared to the traditional FxLMS, the attenuation degree is lower, however the silent point is created at a position $2 \mathrm{~cm}$ away from the error microphone.

In the next experiment, the desired silent point is located $4 \mathrm{~cm}$ away from the error microphone. Again, the noise power at the remote location is calculated by Eq. (13). The result is plotted in Fig. 8. As seen, the noise power at the remote location is attenuated by about $19 \mathrm{~dB}$ in steady state conditions.

The simulation experiments discussed above can be repeated for other values of $r$. The result can be then plotted as shown in Fig. 9. As seen, by increasing the distance between the error microphone and the desired remote silent point the performance of the ANC system (attenuation level) is degraded. When the distance exceeds a particular level, the attenuation degree becomes zero (or even negative). This means that the proposed Remote FxLMS algorithm is only able to create a silent point in a limited range from the error microphone.

\section{CONCLUSION}

Traditional FxLMS-based ANC can only create silence at the location of the error microphone. This causes ineffective use of space available in the quiet zone that is produced around the silent point. The proposed Remote FxLMS algorithm is able to create silence in a location far from the error microphone. The error microphone is still used by the Remote FxLMS algorithm; however, the silent point is created in another location. The construction of the proposed system can be achieved through a real-time adaptation process that is proposed in the paper (Remote FxLMS algorithm). Simulation results show that the proposed system is efficient for creating silent point in an acceptable range from the error microphone.

\section{REFERENCES}

[1] S. M. Kuo and D. R. Morgan, Active noise control systems: algorithms and DSP implementations. New York, NY, USA: Wiley Interscience, 1996.

[2] P. Filippi, D. Habault, J. P. Lefevre, and A. Bergassoli, Acoustics, basic physics, theory and methods. London, UK: Academic Press, 1999, ch. 1.

[3] J. Yuan, "A relaxed condition for "perfect" cancellation of broadband noise in 3d enclosures," The Journal of the Acoustical Society of America, vol. 107, no. 6, pp. 3235-3244, 2000.

[4] B. J. Zimmer, S. P. Lipshitz, K. A. Morris, J. Vanderkoy, and E. E. Obasi, "An improved acoustic model for active noise control in a duct," Journal of Dynamic Systems, Measurement, and Control, pp. 382-395, 2003.

[5] H. Lim, S. V. Utyuzhnikov, Y. W. Lam, and A. Turan, "Multi-domain active sound control and noise shielding," The Journal of the Acoustical Society of America, vol. 129, no. 2, pp. 717-725, 2011.

[6] S. J. Elliott, Signal Processing for Active Control. San Diego, CA.: Academic Press, 2001, ch. 2.

[7] J. C. Burgess, "Active adaptive sound control in a duct: computer simulation," Journal of the Acoustical Society of America, vol. 70, pp. 715-726, 1981.

[8] G. E. Warnaka, L. A. Poole, and J. Tichy, "Active acoustic attenuators," U.S. Patent 4473906, 1984

[9] B. Widrow, D. Shur, and S. Shaffer, "On adaptive inverse control," in Proceeding of the 15th Asilomar Conference on Circuits, Systems and Computers, Nov. 1981, pp. 185-189.

[10] I. Tabatabaei Ardekani and W. H. Abdulla, "Theoretical convergence analysis of FxLMS algorithm," Signal Processing, vol. 90, no. 12, pp. $3046-3055,2010$.

[11] - "On the convergence of real-time active noise control systems," Signal Processing, vol. 91, no. 5, pp. 1262-1274, 2011.

[12] _ "On the stability of adaptation process in active noise control systems," Journal of Acoustical Society of America, vol. 129, no. 1, pp. 173-184, 2011.

[13] _ _ "Effects of imperfect secondary path modeling on adaptive active noise control systems," IEEE Transactions on Control Systems Technology, vol. 20, no. 5, pp. $1252-1262$, sept. 2012.

[14] - "Root locus analysis and design of the adaptation process in active noise control," Journal of Acoustical Society of America, vol. 132, no. 4, 2012. 